\title{
ALL YOU NEED IS HIGHER ACCURACY? ON THE QUEST FOR MINIMUM ACCEPTABLE ACCURACY FOR MEDICAL ARTIFICIAL INTELLIGENCE
}

\author{
Federico Cabitza ${ }^{1}$, Andrea Campagner ${ }^{1}$, Francesco Del Zotti ${ }^{2}$, Alice Ravizza ${ }^{3}$ \\ and Federico Sternini ${ }^{3}$ \\ ${ }^{1}$ University of Milano-Bicocca, Viale Sarca 336, 20126, Milano, Italy \\ ${ }^{2}$ NetAudit, Verona, Italy \\ ${ }^{3}$ USE-ME-D srl, I3P Politecnico di Torino, Corso Castelfidardo, 30/a, 10129, Torino, Italy
}

\begin{abstract}
In this paper we will discuss the concept of the minimum level of accuracy an Artificial Intelligence system must exhibit in medical settings to be fit to its intended use and improve the daily practice of its intended users, the medical doctors. We will consider simple binary classification tasks in both diagnostic and prognostic ambit (like to discriminate between normal/abnormal case, and improvement/no improvement prospects). We will make the point that the common ways to determine this minimum acceptable accuracy are fraught with many conceptual and practical troubles. We will report about a small user study conducted to elicit the discriminative requirements from a sample of medical doctors, stratified both in general practitioners and specialists. Finally, we will present a simple nomogram by which to determine the minimum accuracy of a technological aid, once the human average performance and the desired level of accuracy are known. The nomogram is to be intended as a provocative simple tool to recognize that the technological tool is less important than a sound protocol in which to use it, responsibly and paying due attention to the role of the human decision makers.
\end{abstract}

\section{KEYWORDS}

Medical Artificial Intelligence, Minimum Acceptable Accuracy, Validation, Healthcare

\section{INTRODUCTION}

In this paper we focus on a concept that is seemingly straightforward to understand, the "Minimum Acceptable Accuracy" (MAA) that a classification system must exhibit in medical settings, but that nevertheless is difficult to define. Setting a specific MAA can be important for at least three broad purposes: for verification and validation of a Medical Artificial Intelligence (MAI); for certification purposes of the products embedding such a MAI; and also to allow for a reasonable accuracy - generalization trade-off, which parallels the known bias - variance trade-off at the point-of-care (Belkin et al., 2019). In particular, this latter concern refers to the fact that, once a system has achieved a required MAA, it could be counterproductive to train it further, as increases in accuracy could actually be associated with some sort of statistical overfitting, and hence with lower performance whenever the "best" model were fed with instances that are different from the ones used for training and in the validation phase. Not to mention that a MAI that greatly exceeds the required level of accuracy could induce stronger over-reliance on its capabilities and mislead its users (paradoxically) more often than a less accurate system of which the end users are a little wary of (we will come back to this point later on, when speaking of automation bias and complacency).

A MAA threshold can be set in mainly three ways, of which the first two are the most common ones: first, by asking the experts (including the prospective users, that is the medical doctors who are supposed to make decision with the MAI support); then, by considering previous performance as a benchmark (both of human decision makers and previous existing MAI systems). We will briefly discuss these two broad methods, and refer to two studies that we performed in this mold. Lastly, we will also propose a third approach, grounding on a new analytical way to assess MAA, and we will introduce a nomogram to help set its value graphically. This nomogram is a two-dimensional diagram that allows to calculate a probabilistic function yielding the required MAA of a MAI in a prototypical business use case. 


\section{A SURVEY OF METHODS AND THEIR SHORTCOMINGS}

In this section we will briefly review three ways to focus on the concept of acceptability regarding the predictive performance of a Machine Learning (ML) model to be deployed in a medical context.

\subsection{Benchmarking with Similar Systems}

As we mentioned above, a common, bottom-up, approach to define acceptability in regard to the predictive performance of a Machine Learning (ML) model is to rely on the community of ML practitioners, and take them as sort of arbiters: according to this common-sense view, it is acceptable the performance that the community (including both the authors of a study and its peer reviewers) consider to be worthy of publication in the specialist literature. This translates in the comparing of the performance of a new ML model to the performance of models that had been previously developed for the same medical task: the MAA is then associated with a performance that is at least equal, if not superior, to that of existing decision support aids, the so called "state of the art".

In (Sternini et al., 2020), we reviewed 49 recent articles reporting the MAI performance for three diagnostic tasks for health conditions of different severity, namely: psoriasis, knee osteoarthritis and breast cancer. As expected, we found great differences in the average scores among the medical tasks (from breast cancer applications, average accuracy $76 \%$, to the psoriasis detection ones, $86 \%$ ) but, more surprisingly yet, also high variability within the single tasks (e.g., $28 \%$ and $21 \%$ were the observed standard deviations of the average scores for breast cancer and psoriasis, respectively). Although of limited scope, our review shows how nearly 2 applications out of 3 could exhibit performances as good as that of oracles (nearly 100\%) and as poor as that of systems that just guess (nearly $50 \%$ ).

Thus, although this comparative approach is straightforward and seemingly effective, here we make the point that it is also inadequate. First of all, accuracy-related measures (ranging through sensitivity, specificity, area under the curve, accuracy, $F_{1}$ score, et cetera) are only seemingly comparable with each other across different studies: strictly speaking these metrics can be compared only if related to the same "test set", so that it is possible to speak of models that support the same discriminative task. If we intended that different test sets for the same discriminative task have to be considered random samples drawn from the same population of similar cases (an assumption that is difficult to consider fully applicable), then comparisons can yield statistically significant results only if confidence intervals are provided and these are computed verifying the underlying assumptions on the nature of the distribution: however, reporting performance metrics with their confidence intervals is still not a widespread and common practice.

Thus, even if we assumed two studies comparable when pertaining to the same "medical task" (like "detecting diabetic retinopathy"), the current lack of reporting standards (the guidelines known as TRIPOD-ML, CONSORT-AI and SPIRIT-AI are still in development (Liu et al., 2019a)) often makes the comparison of models' skills an arbitrary and vague endeavour. Moreover, even if we accepted that the literature could provide reliable benchmarks, comparison with other tools cannot tell whether the new system is really acceptable in absolute terms, but only that it is better than other, possibly inadequate, tools.

\subsection{Benchmarking with Humans}

To overcome the objection just mentioned, another common approach to setting MAA requires to relate it to the average accuracy of human doctors performing the same discriminative task: the reasoning here is that if doctors, after extensive training and sufficient experience, are considered adequate to the task, no one should expect an automatic system perform better than them: and when it does (as often reported in the recent specialist literature, e.g., (Liu et al., 2019b)) big headlines on the news are guaranteed too.

In the literature review mentioned above (Sternini et al., 2020), we also observed that comparing the machine performance with that of humans on the same instance is relatively, and surprisingly, common (especially considering the additional effort required to involve professionals with usually very busy schedules, like doctors are), and it was done in 13 studies out of 49 (27\%), i.e., in slightly more than one quarter of the survey sample. 
More generally speaking, we could be interested in understanding what the average human baseline is, so that we can set acceptability right at that level. To this aim, in the literature there are many studies aimed at assessing "the incidence of error rate" in medicine. Among the most-cited studies, (Graber, 2013) reviews several previous contributions and estimates diagnostic human accuracy at $85 \%-87 \%$, while in the radiological domain estimations ranges from approximately $80 \%$ (Bruno et al., 2015) to even more pessimistic estimations, up to just 70\% (Berlin, 2014).

However, although setting human performance as a benchmark could sound reasonable, estimating any average rate can be difficult, or even unfeasible: first of all, human accuracy does not only depend on the specific medical task, which in its turn depends on the health condition at hand and the investigation tool (e.g., MRI, CTs, X-rays, lab exams); but also on idiosyncratic aspects, like the complexity, severity and rarity of the case (for instance, (Semigran et al., 2016) report that doctors are better in high-acuity (vs low-acuity cases) and uncommon cases (vs common cases)); and on characteristics of the work setting (like throughput, time constraints (ALQahtani et al., 2016), work shift policies (Hanna et al., 2018), modality brand and age), not to mention the doctors involved, their expertise mix, and even their states of mind. Thus, relying on the thaumaturgical value of averages on a sufficiently large sample (which is seldom the case), could not be enough to assess an average accuracy threshold above which to set the MAA in a less than highly situated and local manner.

Furthermore, the merely discriminative task for which the error rate is measured could be just an abstraction of a more complex interpretative task, from which it cannot be decoupled unless in a fictional, laboratory setting (e.g., radiologists do not only look at and classify images, but also consult the patient record and consider the needs and hypotheses of the referring specialist). In particular, also a "laboratory effect" has been observed (Gur et al., 2008): this is a somehow counterintuitive phenomenon by which doctors are more likely to perform worse in controlled experimental conditions, even if these create an ideal situation of uninterrupted concentration, than when they actually work at the hospital, and their decision can affect the health of real patients. If this effect is common as conjectured, researchers performing MAI-humans comparisons on retrospective data could seriously underestimate the actual doctors' performance.

That said, even assuming the estimate correct, and setting the MAA slightly above the average human performance, we should consider the following point: can the introduction of a decision aid in a hospital be assimilated to the entry of a new member into an existing team of doctors? If this were the case, would it be right to bear the investment necessary for acquiring a technological member, and the related operating costs, when this latter one is roughly worse than half the other members?

\subsection{Asking the Experts}

Another simple way to set the MAA threshold requires to rely on the experts' opinion. As arbitrary as this approach might seem, we also will give our opinion on what the MAA should be at the end of this contribution. However, few of these opinions can be found in the specialist literature. Combining the two approaches mentioned above, (Floares, 2009) sets MAA at about $80 \%$ grounding on both "the performances of the best commercial systems" and the fact that "the majority of physicians [involved in his study] will agree that the prediction accuracy should be at least $80 \%$ ".

We wanted to replicate this claim and performed a simple user study by asking a convenience sample of specialists and family doctors what the MAA for a MAI should be in both a generic diagnostic and prognostic task (i.e., respectively, in predicting the patient's health problem and in predicting the patient's outcome after treatment). Forty-eight (48) General Practitioners and 31 specialists (mainly orthopedists) participated, by filling-in a two-question online questionnaire where we explicitly asked "What is, in your opinion, the maximum error rate (in percentage) of the system that you believe is tolerable for a MAI system to be practically useful to you in your daily work?": on average the general practitioners indicated a diagnostic MAA of $88 \%$ (median: $93 \%$ ) and a prognostic MAA of $83 \%$ (median: $88 \%$ ). On the other hand, the specialists were harder to please (not totally surprisingly) and expressed a MAA of 95\% (median: 94\%) and 90\% (median: $90 \%$ ) for diagnostic and prognostic purposes, respectively.

In particular, with reference to Figure 1, we can notice that the differences between general practictioners (GPs) and specialists are not statistically significant, while the difference between the minimum acceptable diagnostic and prognostic performance reported by the specialists is statistically significant (i.e., no overlap occurs between the confidence intervals of the medians). 


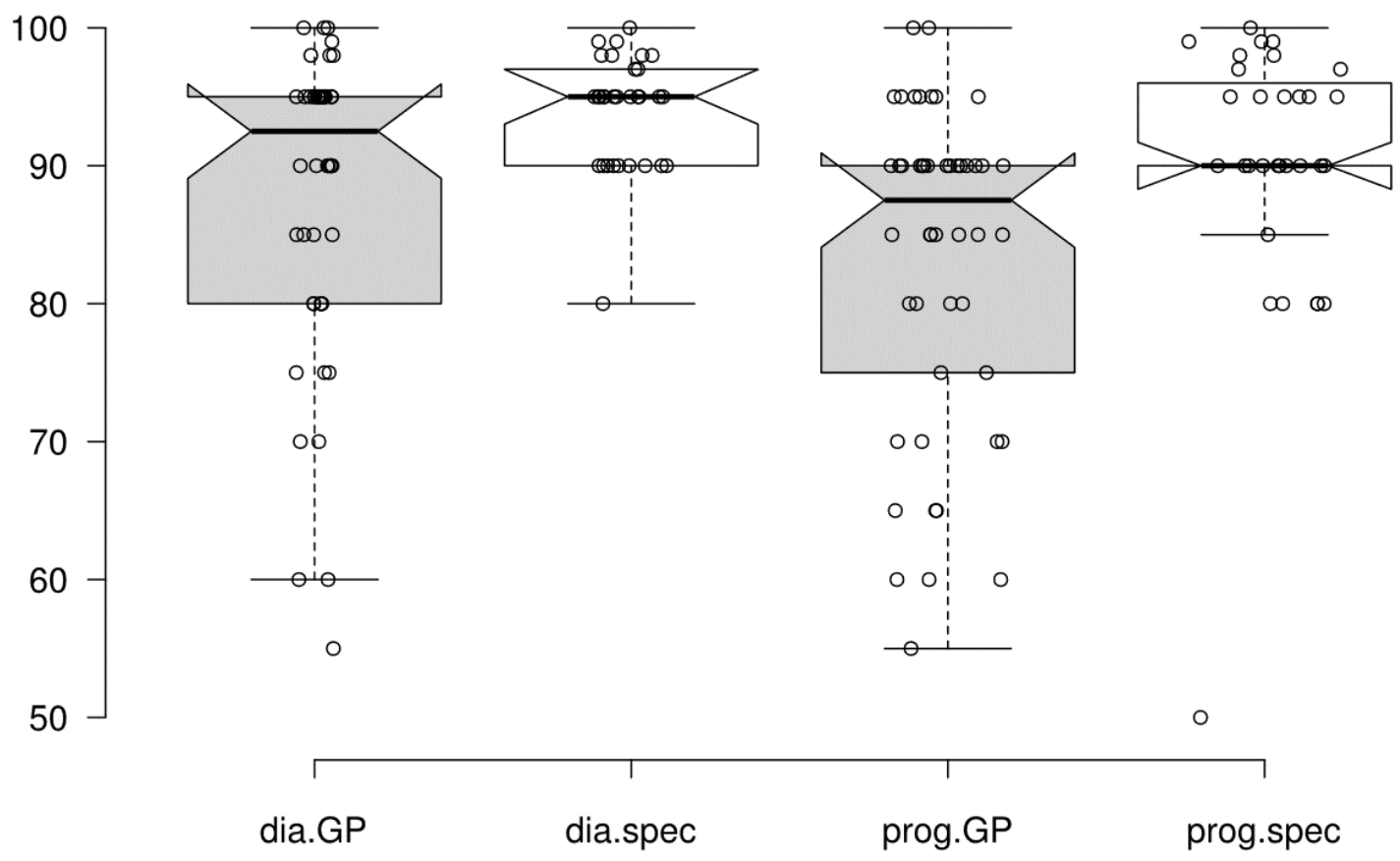

Figure 1. Minimum Acceptable Accuracy, as it is perceived by a sample of General Practitioners (GP, N=48) and Specialists (spec, $\mathrm{N}=31$ ) for diagnostic (dia) and prognostic (prog) purposes

Asking the key opinion leaders, or just the prospective users of the system to be validated, is a sound principle of the requirement engineering practice. However, just asking "how much accurate users want their decision support to be" could be not sufficient to improve their practice. First, doctors could overestimate their own accuracy, and hence be too much demanding with respect to their decision aids, and end up by asking for unrealistically (and unnecessarily) accurate tools if their main aim is the improvement of doctors' performance, especially in terms of effectiveness (rate of correct and appropriate decisions) and ultimately, the improvement of the patients' outcome. Moreover, even if doctors were warned of their average performance (at least in terms of approximate estimate, as this can be drawn from the literature as mentioned above), one should not expect they can understand how the accuracy of a decision tool could impact the overall team performance, as this aspect has still to be investigated in the specialist literature.

\subsection{Looking at How Teams Work (or the Protocol-Based Approach)}

All of the above methods, although very common and sounding perfectly reasonable at first glance, suffer from both minor and major flaws. The most important flaw, in our view, is that they subsume an idea of decision support that is disembodied and abstracted away from the situated ways in which a decision aid is actually used in real-world practice. In the next future, and probably even in the long term, no MAI will be supposed to provide a diagnosis or formulate a prognosis alone, since these tasks will continue to be well under the human responsibility, also (but not only) because these latter ones will get access to many more elements (including the psychological, emotional, social ones) than those can be properly digitized, and hence made available to the machine's scrutiny.

Likewise, users could find difficult to understand what kind of support they need to improve their own performance, as they could underestimate how their expectations, biases and prejudices could affect their decisions, when these are made in light of the machine's recommendations: suffice it here to mention the effects known as "automation bias" (Goddard et al., 2012), that is over-relying on the machine's advice believing that it will never (or very seldom) be wrong, "automation complacency" (Lyell and Coiera, 2016), that is when one overestimate their capability to understand when the machine is wrong or it should raise an alarm, and the "prejudice against the machine"(Cabitza, 2019), which is a sort of opposite attitude observed in a cardiological 
study, when the participants trusted more a simulated human colleague than a MAI although they were proposing the same wrong options.

For all these reasons, we propose a further approach to determine MAA, which can be called analytical but also protocol-based, because we can trace it back to a reasonably common, and realistic, business scenario of MAI use. In this case, one could think of a typical point-of-care use case, where the medical AI is used by one doctor to be advised about a case. This setting is coherent with the so called centaur model (Goldstein et al., 2017), that is a model proposed in medical decision making in which humans and machines team up in sort of augmented, hybrid teams that collaborate to make a decision and where every member of the team does what they excel at the most: intuition, empathy, communication the human part (represented as the head and heart of the centaur), big data mining, logical reasoning, trend detection and pattern matching the computational part (i.e., the muscles and legs of the centaur). In most of the cases, the machine would just confirm and corroborate the doctor's interpretation, even in those (hopefully few) cases that both the human and the MAI were wrong (in this case, the final decision by the doctor could easily be affected by a technologically-augmented, if not induced, confirmation bias). However, in a certain number of cases, doctors and AI will disagree. In that case, a safe protocol would then require the involvement of a third agent, a second human doctor acting as an arbiter, who could re-read the available imaging (or evidence) available for the case at hand and express a sort of second (or actually third) opinion. In the simplest case, that is a binary task, the third opinion would just confirm one diagnostic hypothesis and automatically outvote the other. Although a final decision could be taken by majority vote, a deeper interaction between the human raters is probably as much an inefficient further step as an advisable one in the protocol to achieve full accountability.

If we take this simple case as a basis for our centaur protocol, we can show that the analytical expression of the formula giving the accuracy of the technology-supported agency can easily be derived. First of all, we adopt the simplifying assumption that the accuracy of the human raters is known and is equal to a constant number acci. Then, the formula that represents the probability that the majority decision of the group of three raters (thus, including the $\mathrm{AI}$ ) is indeed the correct one (under the assumption that the raters' opinions are independent or can be requested to be so) is given by the following formula:

$$
\sum_{k=2}^{3} \sum_{R \in R_{k}} \prod_{r_{i} \in R} a c c_{i} \prod_{r_{j} \in R}\left(1-a c c_{j}\right)
$$

In this formula (which follows from a simple combinatorial argument) $R_{k}$ is the set of groups of raters of size $\mathrm{k}$, acc $_{\mathrm{i}}$ is the accuracy of rater $\mathrm{i}$.

As we previously mentioned, this formula can then be used to determine an analytical expression for the MAA of a MAI which is to support the other 2 human raters. Indeed, let us suppose that the two human doctors have an average accuracy of $85 \%$ and we want to establish a team that is augmented with a MAI and we want to determine the MAA for this MAI so that the overall accuracy of the augmented team is higher than the accuracy of the single human doctors, and is at least $90 \%$. The MAA can then be found by solving an inequality in which the previous formula is set as greater than our desired group accuracy (I.e. 90\%) and the only parameter to be determined is the accuracy of the MAI. It could then seem counterintuitive to notice that to satisfy such a requirement and guarantee an overall accuracy of $90 \%$, only a $70 \%$-accurate $\mathrm{AI}$ is needed!

For the sake of reference, we translate this analytical expression into a diagram, or better yet, a nomogram. By means of such a nomogram (depicted in Figure 2) it is possible to graphically determine the MAA of a MAI, given the accuracy of the two human doctors, and the desired level of accuracy of the whole "hybrid team", which is augmented by the MAI and adopts the simple majority voting strategy to settle case of discordance. The example depicted in the nomogram reported in Figure 2 represents the above described example scenario.

Obviously, the nomogram can be read also the other way round, by drawing a broken line from the vertical axis on the right up to the colored segment and then down up to the horizontal axis: so, if we use an $90 \%$ accurate MAI within a team where it is used under the engagement protocol described above, the performance of a team of doctors who are $85 \%$ accurate, would be improved up to $95 \%$. 


\section{Al Minimum Acceptable Accuracy}

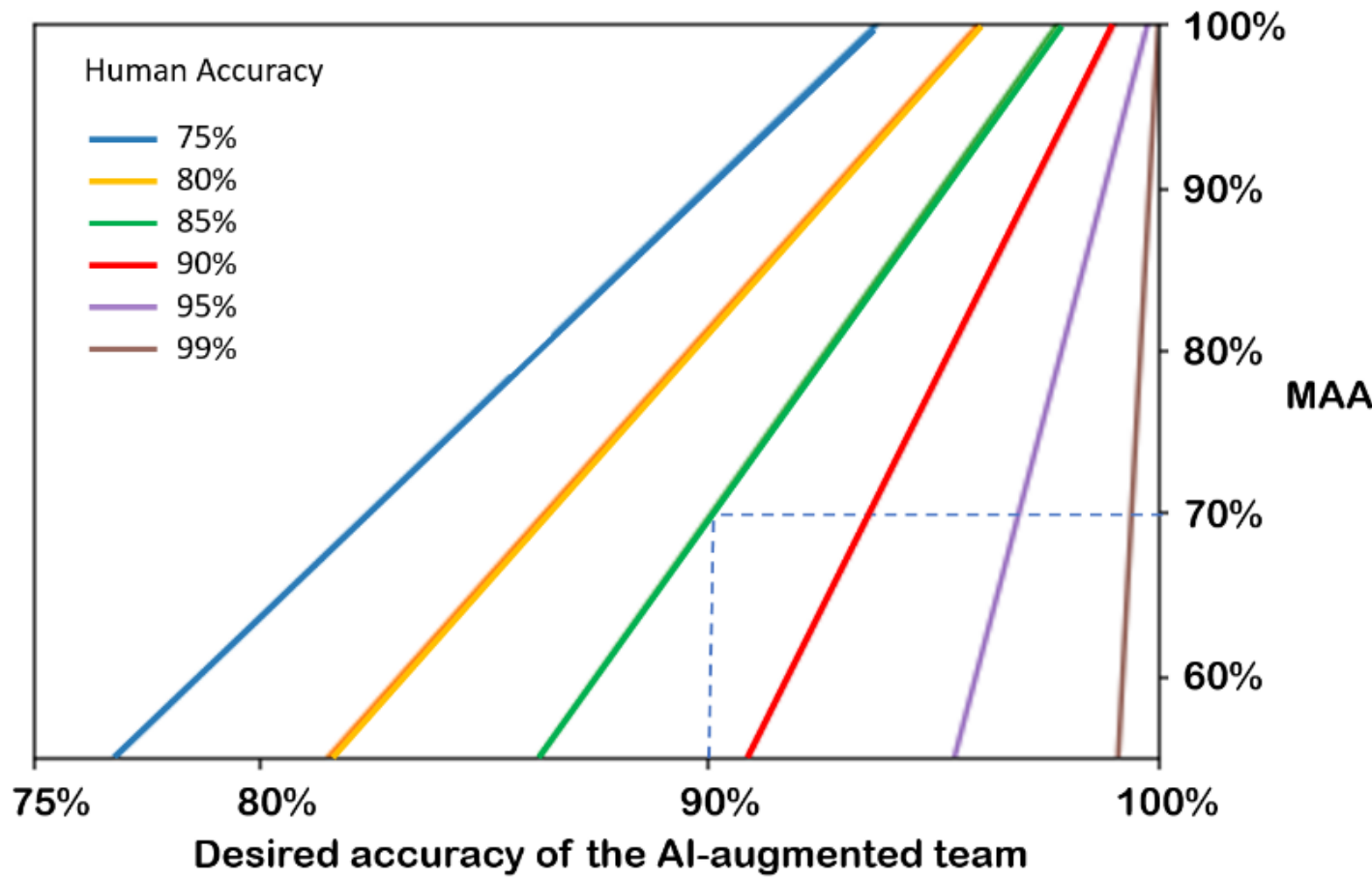

Figure 2. The nomogram to determine the minimum acceptable accuracy (MAA) of an AI in medical context. The dashed line suggests how to use the nomogram to read the MAA on the vertical line, once the desired accuracy of the medical team has been set, knowing the accuracy of the human members (colored segment)

Thus, this analytical approach allows to the determine of the MAA of a MAI (and the related illustrative nomogram) and also shows how the technological tool (I.e. the MAI), and its accuracy, in itself could be less important than the interaction protocol in which the MAI is considered: indeed, as we previously shown, a 70\% accurate MAI may be in some cases sufficient to set up an interaction protocol that is more accurate than the sum of its parts.

\section{CONCLUSIONS}

In this paper we have outlined the case of determining a minimum threshold according to which to consider the performance of an Artificial Intelligence - AI (applied to discriminative and classification tasks) sufficiently valid in medical settings: we called such a threshold, the Minimum Acceptable Accuracy (MAA) a valid medical AI (MAI) system must exhibit to be such. Although it is easy to convince ourselves that such a concept is important and defining a MAA in Medical decision supports (one for each medical task or health condition) is the first step to understand if (buying) and adopting such an aid is necessary to improve medical practice, we also shed light on the fact that the common ways to understand if a MAI is accurate enough to be fit to its intended use (that is to support and improve doctors in their daily tasks) are fraught with simplistic assumptions or over-ambitious estimates.

We also reported the results of a small user study, in which we asked 79 medical doctors "how much accurate" they would require their MAI to be to perceive it as useful for their practical aims; and then we presented a simple nomogram by which to determine the MAA in a task-agnostic manner, when the accuracy of the human doctors is known (or assumed) and the accuracy requirement is adopted at the higher level, that is the level of the hybrid human-machine team where the MAI is adopted within a usage protocol that is aimed at finding the appropriate trade-off between efficiency (e.g., faster decisions), effectiveness (e.g., higher 
accuracy) and accountability (e.g., humans have the final say and can discuss controversial cases). This contribution is also aimed at addressing the provocative question mentioned in the title with a reflective attitude. In fact, we have also shown how doctors, if invited to express their requirements, would probably exaggerate the importance of relying on accurate decision aids to have their practice improved, and would push for more and more accurate tools (which nevertheless could facilitate the emergence of harmful attitudes like automation bias and complacency).

The nomogram we devised is then to be intended more as an informative simple tool, than an actual decision aid, to recognize that the technological tool is less important than a sound protocol in which to use it, both responsibly and by paying due attention to the role of the human decision makers. In fact, medicine has always been a collaborative practice, and a simple protocol can make up the shortcomings of technology and the limits of any single human by simply fostering discussion and making a technologically-augmented support better than the sum of its parts and better than each of the single parts.

\section{ACKNOWLEDGEMENT}

The authors acknowledge that the GP study was conducted on the basis of a sample of data coming in part from a survey conducted by INTRAMED COMMUNICATIONS SRL on a sample of doctors who had been invited to participate in an ECM event organized by this company. The authors are also grateful to the anonymous General Practitioners who accepted the invitation to participate in the survey sent by FDZ, as well as the anonymous specialists who participated to the same survey from the IRCCSS Istituto Ortopedico Galeazzi, Milano, Italy.

\section{REFERENCES}

Al Qahtani, D.A., Rotgans, J.I., Mamede, S., Al Alwan, I., Magzoub, M.E.M., Altayeb, F.M., Mohamedani, M.A., Schmidt, H.G., 2016. Does Time Pressure Have a Negative Effect on Diagnostic Accuracy?. Acad. Med. 91, 710-716. https://doi.org/10.1097/ACM.0000000000001098

Belkin, M., Hsu, D., Ma, S., Mandal, S., 2019. Reconciling modern machine-learning practice and the classical bias-variance trade-off. Proc. Natl. Acad. Sci. 116, 15849-15854. https://doi.org/10.1073/pnas.1903070116

Berlin, L., 2014. Radiologic errors, past, present and future. Diagnosis 1, 79-84. https://doi.org/10.1515/dx-2013-0012

Bruno, M.A., Walker, E.A., Abujudeh, H.H., 2015. Understanding and Confronting Our Mistakes: The Epidemiology of Error in Radiology and Strategies for Error Reduction. RadioGraphics 35, 1668-1676. https://doi.org/10.1148/rg.2015150023

Cabitza, F., 2019. Biases Affecting Human Decision Making in AI-Supported Second Opinion Settings, in: Torra, V., Narukawa, Y., Pasi, G., Viviani, M. (Eds.), Modeling Decisions for Artificial Intelligence. Springer International Publishing, Cham, pp. 283-294.

Floares, A.G., 2009. Using computational intelligence to develop intelligent clinical decision support systems, in: International Meeting on Computational Intelligence Methods for Bioinformatics and Biostatistics. Springer, pp. 266-275.

Goddard, K., Roudsari, A., Wyatt, J.C., 2012. Automation bias: a systematic review of frequency, effect mediators, and mitigators. J. Am. Med. Inform. Assoc. 19, 121-127. https://doi.org/10.1136/amiajnl-2011-000089

Goldstein, I.M., Lawrence, J., Miner, A.S., 2017. Human-Machine Collaboration in Cancer and Beyond: The Centaur Care Model. JAMA Oncol. 3, 1303. https://doi.org/10.1001/jamaoncol.2016.6413

Graber, M.L., 2013. The incidence of diagnostic error in medicine. BMJ Qual. Saf. 22, ii21-ii27. https://doi.org/10.1136/bmjqs-2012-001615

Gur, D., Bandos, A.I., Cohen, C.S., Hakim, C.M., Hardesty, L.A., Ganott, M.A., Perrin, R.L., Poller, W.R., Shah, R., Sumkin, J.H., Wallace, L.P., Rockette, H.E., 2008. The "Laboratory" Effect: Comparing Radiologists' Performance and Variability during Prospective Clinical and Laboratory Mammography Interpretations. Radiology 249, 47-53. https://doi.org/10.1148/radiol.2491072025

Hanna, T.N., Lamoureux, C., Krupinski, E.A., Weber, S., Johnson, J.-O., 2018. Effect of Shift, Schedule, and Volume on Interpretive Accuracy: A Retrospective Analysis of 2.9 Million Radiologic Examinations. Radiology 287, $205-212$. https://doi.org/10.1148/radiol.2017170555 
Liu, X., Faes, L., Calvert, M.J., Denniston, A.K., 2019a. Extension of the CONSORT and SPIRIT statements. The Lancet 394, 1225. https://doi.org/10.1016/S0140-6736(19)31819-7

Liu, X., Faes, L., Kale, A.U., Wagner, S.K., Fu, D.J., Bruynseels, A., Mahendiran, T., Moraes, G., Shamdas, M., Kern, C., others, 2019b. A comparison of deep learning performance against health-care professionals in detecting diseases from medical imaging: a systematic review and meta-analysis. Lancet Digit. Health 1, e271-e297.

Lyell, D., Coiera, E., 2016. Automation bias and verification complexity: a systematic review. J. Am. Med. Inform. Assoc. ocw105. https://doi.org/10.1093/jamia/ocw105

Semigran, H.L., Levine, D.M., Nundy, S., Mehrotra, A., 2016. Comparison of Physician and Computer Diagnostic Accuracy. JAMA Intern. Med. 176, 1860. https://doi.org/10.1001/jamainternmed.2016.6001

Sternini, F., Ravizza, A., Cabitza, F., 2020. How accurate do you want it? Defining Minimum Required Accuracy for Medical Artificial Intelligence, in: To Be Published. 